\title{
PERSISTÊNCIA DE AGROTÓXICOS INDICADOS NA PRODUÇÃO INTEGRADA DE PÊSSEGO A Trichogramma pretiosum RILEY, 1879 (HYMENOPTERA: TRICHOGRAMMATIDAE) ${ }^{1}$
}

\author{
FABRIZIO PINHEIRO GIOLO ${ }^{2}$, ANDERSON DIONEI GRÜTZMACHER ${ }^{3}$, CRISTIANE GINDRI MANZONI², \\ JOSÉ CARLOS FACHINELLO ${ }^{4}$, DOUGLAS DANIEL GRÜTZMACHER ${ }^{5}$, SANDRO DANIEL NÖRNBERG ${ }^{6}$
}

RESUMO - A persistência (duração da atividade nociva) de cinco agrotóxicos indicados na Produção Integrada de Pêssego (PIP) foi avaliada, expondo-se adultos de Trichogramma pretiosum Riley, 1879 (Hymenoptera: Trichogrammatidae) ao contato com resíduos de inseticidas, pulverizados sobre folhas, a diferentes intervalos de tempo, baseando-se na metodologia sugerida pela International Organization for Biological and Integrated Control of Noxious Animals and Plants (IOBC). Os resultados obtidos em relação à persistência, permitiu classificar os agrotóxicos, produto comercial/ingrediente ativo (g ou mL de produto comercial 100L $\mathrm{L}^{-1}$ ), Dipterex 500/triclorfom (300), Sumithion $500 \mathrm{CE} /$ fenitrotiona (150) e Tiomet $400 \mathrm{CE} /$ dimetoato (120) como levemente persistentes (5-15 dias); o inseticida Malathion $1000 \mathrm{CE} /$ malationa (200) como moderadamente persistente (16-30 dias) e o fungicida/acaricida Kumulus DF/ enxofre (600) como persistente ( $>31$ dias) a adultos de T. pretiosum.

Termos para Indexação: controle biológico, controle químico, parasitóide de ovos, seletividade a inimigos naturais.

\section{PERSISTENCE OF PESTICIDES USED IN INTEGRATED PEACH PRODUCTION ON Trichogramma pretiosum RILEY, 1879 (HYMENOPTERA: TRICHOGRAMMATIDAE)}

\begin{abstract}
The persistence (duration of harmful activity) of five insecticides indicated in the Integrated Peach Production (IPP) was evaluated by adult exposure of Trichogramma pretiosum Riley, 1879 (Hymenoptera: Trichogrammatidae) to insecticide residues on plant leaves at different time intervals after treatment using the International Organization for Biological and Integrated Control of Noxious Animals and Plants (IOBC) based on the suggested methodology.The results obtained in relation to the disease persistence will permit to classify the pesticides, commercial formulation/active ingredient ( $\mathrm{g}$ or mL commercial formulation 100L $\mathrm{L}^{-1}$ ), Dipterex 500/ trichlorphon (300), Sumithion $500 \mathrm{CE} /$ fenitrothion (150) and Tiomet $400 \mathrm{CE} /$ dimethoate (120) as slightly persistent (5-15 days); the insecticide Malathion $1000 \mathrm{CE} /$ malathion (200) as moderately persistent (16-30 days) and the fungicide Kumulus DF/sulphur (600) as persistent ( $>31$ days) to T. pretiosum adults.
\end{abstract}

Index Terms: biological control, chemical control, egg parasitoids, side-effects to natural enemies.

\section{INTRODUÇÃO}

A cultura do pessegueiro pertence ao grupo de espécies frutíferas que possuem normas técnicas específicas (NTE) para a produção integrada, no projeto denominado Produção Integrada de Pêssego (PIP) (Fachinello et al., 2003), que visa, dentre outros objetivos, à utilização de agrotóxicos seletivos, associando os métodos químico e biológico, para o controle de pragas. Na cultura do pessegueiro, a grafolita, Grapholita molesta (Busck, 1916) (Lepidoptera: Tortricidae), é considerada uma das principais espécies-praga (Botton et al., 2001), sendo o controle químico o principal método utilizado pelos persicultores para reduzir a densidade populacional dessa espécie.

$\mathrm{O}$ alto custo socioeconômico dos inseticidas tradicionais vem impulsionando a busca de alternativas eficientes e ecologicamente compatíveis com o sistema PIP, como, por exemplo, a viabilização do controle biológico. O controle biológico da G. molesta com Trichogramma pretiosum Riley,
1879 (Hymenoptera: Trichogrammatidae) vem sendo estudado em Pelotas, Rio Grande do Sul (Garcia et al., 2006), e demonstra a importância desse agente biológico em um futuro programa de controle biológico aplicado. Além disso, Trichogramma é considerado como indicador, em estudos de seletividade de agrotóxicos, servindo como referência para outros macro e microimenópteros (Hassan, 1998). No entanto, a utilização desses parasitóides, na cultura do pessegueiro, ainda apresenta várias limitações, sendo a carência de informações sobre a seletividade dos agrotóxicos utilizados nessa cultura a organismos benéficos, uma delas. Com relação aos estudos de seletividade na cultura do pessegueiro, Grützmacher et al. (2004) avaliaram alguns agrotóxicos recomendados na PIP sobre a espécie-padrão da "International Organization for Biological and Integrated Control of Noxious Animals and Plants, West Palaeartic Regional Section" (IOBC/WPRS), Trichogramma cacoeciae Marchal, 1927 (Hymenoptera: Trichogrammatidae), realizando vários estudos com adultos e imaturos, incluindo testes de persistência biológica. Posteriormente, Giolo et al. (2005) também avaliaram a

\footnotetext{
'Trabalho 067-07). Recebido em: 20-03-2007. Aceito para publicação em: 18-10-2007. Realizado com apoio financeiro do CNPq e da FAPERGS.

${ }^{2}$ Eng. Agr., Dr. Depto. de Fitossanidade/FAEM/UFPel. "e-mail: fgiolo.faem@hotmail.com.

${ }^{3}$ Eng. Agr., Dr., Prof. Depto. de Fitossanidade/FAEM/UFPel. email: adgrutzm@ufpel.tche.br.

${ }^{4}$ Eng. Agr., Dr., Prof. Depto. de Fitotecnia/FAEM/UFPel. e-mail: jfachi@ufpel.tche.br.

${ }^{5}$ Eng. Agr., Dr., Bolsista PRODOC/CAPES, Depto. de Fitossanidade/FAEM/UFPel. email: douglasdanielg@terra.com.br.

${ }^{6}$ Eng. Agr., Mestrando, Depto. de Fitossanidade/FAEM/UFPel. email: sandronornberg@terra.com.br.
} 
seletividade de agrotóxicos indicados na PIP, porém sobre $T$. Pretiosum, e recomendaram a realização de estudos de persistência biológica para aqueles agrotóxicos considerados não-inócuos a adultos do parasitóide, ou seja, com reduções no parasitismo acima de $30 \%$ (classe 1 da IOBC).

Neste contexto, pesquisas que avaliem a persistência (duração da atividade nociva) são importantes, pois agrotóxicos de rápida degradação no ambiente poderiam ser utilizados com sucesso em modernos sistemas de produção, como, por exemplo, a PIP. Dessa forma, o objetivo do presente estudo foi conhecer a persistência de inseticidas indicados na PIP, tendo como referência o estágio adulto de $T$. pretiosum.

\section{MATERIAL E MÉTODOS}

Os trabalhos foram conduzidos nos laboratórios de Biologia dos Insetos, Controle Biológico e de Pesticidas e na casa de vegetação do Departamento de Fitossanidade (DFs) da Faculdade de Agronomia "Eliseu Maciel" (FAEM) da Universidade Federal de Pelotas (UFPel), Pelotas-RS. Os parasitóides, da espécie $T$. pretiosum, utilizados nos experimentos foram oriundos de criação em laboratório, em câmaras climatizadas (temperatura $25 \pm 1{ }^{\circ} \mathrm{C}$, umidade relativa $70 \pm 10 \%$ e fotofase 14 horas), utilizando-se de ovos do hospedeiro Anagasta kuehniella (Zeller, 1879) (Lepidoptera: Pyralidae).

Os bioensaios foram conduzidos em laboratório $\left(25 \pm 1^{\circ} \mathrm{C}\right.$, umidade relativa $70 \pm 10 \%$ e fotofase de 14 horas), expondo-se adultos (estágio mais sensível) de T. pretiosum a resíduos dos agrotóxicos: produto comercial/ingrediente ativo (dosagem em g ou mL 100L ${ }^{-1}$ ) Dipterex 500/triclorfom (300), Kumulus DF/enxofre (600), Malathion $1000 \mathrm{CE} /$ malationa (200), Sumithion $500 \mathrm{CE} /$ fenitrotiona (150) e tiomet $400 \mathrm{CE} /$ dimetoato (120), pulverizados sobre folhas de videira (planta-padrão da IOBC/WPRS). Quando as plantas atingiram $80-100 \mathrm{~cm}$ de altura com, aproximadamente, 15 folhas desenvolvidas, foram pulverizadas com os agrotóxicos, na dosagem máxima recomendada para a cultura do pessegueiro, até o ponto de escorrimento. No tratamento-testemunha, foi pulverizada apenas água destilada. A pulverização foi realizada com pulverizador manual Guarany ${ }^{\circledR}$, com capacidade de $580 \mathrm{~mL}$. Depois de tratadas, as plantas permaneceram à temperatura ambiente, por cerca de três horas, para evaporação do excesso de umidade, formando uma película seca com o tratamento. Semanalmente, os ápices de todas as plantas foram cortados, eliminando as brotações e gemas apicais, para evitar a emissão de novas folhas. Aos 3; 10; 17; 24 e 31 dias após o tratamento das videiras, folhas de cada tratamento foram coletadas de diversas partes das plantas e expostas por cerca de 24 horas para o contato com adultos do parasitóide e posterior oferta de ovos do hospedeiro alternativo, A. kuehniella, para a avaliação do número de ovos parasitados, conforme descrito na metodologia da IOBC/WPRS (Hassan \& Abdelgader, 2001), adaptada para $T$. pretiosum para atender a características biológicas intrínsecas dessa espécie (Giolo et al., 2005).

Inicialmente, todos os resultados foram testados quanto à normalidade (proc univariate). Para variáveis que apresentaram uma distribuição tendendo a normal, foi realizada análise variância (ANOVA), e, quando o valor de $F$ foi significativo $(\mathrm{p}<0,05)$, as médias foram comparadas pelo teste de TukeyKramer (proc glm). As variáveis que não apresentaram distribuição tendendo a normal, foram submetidas ao teste nãoparamétrico de Kruskal-Wallis (proc npar1way). Se significativos, os dados foram classificados (Conover \& Iman, 1981) e as médias classificadas, obtidas, foram comparadas pelo teste de Bonferroni-Dunn $t$ (proc glm). Todas as análises estatísticas foram realizadas através do programa estatístico "SAS Learning Edition" (SAS Institute, 2002) para um nível de significância de 5\% de probabilidade. Além das análises estatísticas convencionais, foram calculados, a redução no parasitismo para cada agrotóxico em relação à testemunha negativa (água destilada). Utilizou-se da fórmula $\mathrm{RP}=\left(1-\mathrm{R}_{\mathrm{t}} / \mathrm{R}_{\mathrm{c}}\right) * 100$, onde RP é a porcentagem de redução no parasitismo, $R_{t}$ é o valor do parasitismo médio para cada produto e $R_{c}$ o parasitismo médio observado para o tratamento-testemunha (negativa). Com base nessas porcentagens de redução, os agrotóxicos foram classificados segundo os índices propostos pela IOBC/WPRS em: 1, inócuo $(<30 \%)$; 2, levemente nocivo (30-79\%); 3, moderadamente nocivo (80-99\%), e 4, nocivo (>99\%). Quando os agrotóxicos reduziram, em dois bioensaios consecutivos, em menos de $30 \%$, o parasitismo de $T$. pretiosum comparado com a testemunha do bioensaio, foram classificados de acordo com a escala de persistência da IOBC/WPRS em: 1 , vida curta ( $<5$ dias); 2 , levemente persistente (5-15 dias); 3 , moderadamente persistente (16-30 dias), e 4, persistente ( $>31$ dias).

\section{RESULTADOS E DISCUSSÃO}

No Bioensaio I, realizado 03 dias após a pulverização (DAP) das videiras, houve diferenças significativas $(\mathrm{K}=20,65 ; \mathrm{p}=0,0009)$ entre os tratamentos. Todos os inseticidas testados: Dipterex 500, Kumulus DF, Malathion 1000 CE, Sumithion 500 CE e Tiomet 400 $\mathrm{CE}$, reduziram significativamente $(\mathrm{p}<0,05$, Bonferroni-Dunn $t$ ) o número de ovos parasitados em relação à testemunha do bioensaio, com valores de 0,$30 ; 1,48 ; 0,00 ; 10,81$ e 0,00 ovos parasitados por fêmea de T. pretiosum, ocasionando reduções no parasitismo de 99,$31 ; 96,58 ; 100,00 ; 75,02$ e 100,00\%, respectivamente. Quando se utiliza a classificação proposta pela IOBC/WPRS, verifica-se, no Bioensaio I, que apenas o inseticida organofosforado Sumithion $500 \mathrm{CE}$, com $75,02 \%$ de redução no parasitismo, foi classificado como levemente nocivo (classe 2), e o fungicida Kumulus DF, com 96,58\% de redução no parasitismo, foi moderadamente nocivo (classe 3). Os demais agrotóxicos, todos inseticidas organofosforados (Dipterex 500, Malathion 1000 CE e Tiomet $400 \mathrm{CE}$ ), foram nocivos (classe 4), pois reduziram acima de $99,00 \%$ o parasitismo de $T$. pretiosum (Tabela 1 ). No Bioensaio II, utilizando folhas de videira com resíduos aos 10 DAP dos tratamentos, também foi observada diferença significativa $(K=17,12 ; \mathrm{p}=0,0043)$ entre os tratamentos. Apenas o inseticida Sumithion $500 \mathrm{CE}$ não diferiu significativamente $(\mathrm{p}>0,05$, Bonferroni-Dunn $t$ ) do tratamento-testemunha negativa (água destilada). Todos os demais agrotóxicos testados: Dipterex 500, Kumulus DF, Malathion 1000 CE e Tiomet 400 CE, diferiram significativamente $(\mathrm{p}<0,05$, Bonferroni-Dunn $t)$ da testemunha 
negativa do bioensaio. O inseticida Malathion $1000 \mathrm{CE}$ foi o que apresentou maior toxicidade, com valor nulo para a variável número de ovos parasitados por fêmea, diferindo significativamente $(\mathrm{p}<0,05$, Bonferroni-Dunn $t)$ da testemunha e também dos inseticidas Dipterex 500 e Sumithon 500 CE. Esses últimos apresentaram valores de 16,86 e 18,25 ovos parasitados por fêmea de T. pretiosum, respectivamente. Os agrotóxicos Kumulus DF e Tiomet 400 CE permitiram, respectivamente, que 4,52 e 14,48 ovos fossem parasitados por fêmea. Atribuindo-se as classes da IOBC/ WPRS, Dipterex 500, Sumithion 500 CE e Tiomet 400 CE foram levemente nocivos (classe 2), com reduções no parasitismo de 49,20; 45,01 e 56,37\%. Por outro lado, Kumulus DF e Malathion $1000 \mathrm{CE}$ foram classificados, respectivamente, como moderadamente nocivo (classe 3) e nocivo (classe 4), reduzindo em 86,38 e $100,00 \%$ o parasitismo de $T$. pretiosum (Tabela 1 ). No Bioensaio III, aos 17 DAP, os tratamentos apresentaram diferença significativa $(\mathrm{K}=18,05 ; \mathrm{p}=0,0029)$. No entanto, apenas os tratamentos Kumulus DF, Sumithion 500 CE e Malathion 1000 CE, com 4,44; 28,48 e 3,01 ovos parasitados por fềmea de T. pretiosum, diferiram significativamente $(\mathrm{p}<0,05$, Bonferroni-Dunn $t)$ da testemunha, onde ocorreram 38,40 ovos parasitados por fêmea. Os inseticidas organofosforados, Dipterex 500 e Tiomet $400 \mathrm{CE}$, com 33,65 e 40,21 ovos parasitados, não diferiram ( $\mathrm{p}>0,05$, Bonferroni-Dunn $t$ ) da testemunha. Aos 17 DAP, os agrotóxicos demonstraram redução do efeito deletério sobre o parasitismo, que foi expressa na classificação atribuída aos produtos. Dessa forma, Dipterex 500, Sumithion $500 \mathrm{CE}$ e Tiomet $400 \mathrm{CE}$ foram considerados inócuos (classe 1), com reduções inferiores a $30,00 \%$. Por outro lado, Kumulus DF e Malathion $1000 \mathrm{CE}$ permaneceram ocasionando efeitos deletérios, respectivamente, de 88,44 e $92,16 \%$, sendo considerados moderadamente nocivos (classe 3) (Tabela 1). No Bioensaio IV, realizado com folhas aos 24 DAP, houve diferença significativa $(\mathrm{F}=4,57 ; \mathrm{gl}=5,17 ; \mathrm{p}=0,0080)$ entre os tratamentos. Porém, apenas o fungicida à base de enxofre, Kumulus DF, afetou significativamente o parasitóide ( $<<0,05$, Tukey-Kramer) com 8,91 ovos parasitados por fêmea, em relação à testemunha do bioensaio, que foi de 28,29 ovos parasitados por fêmea. Nesse bioensaio, apenas o fungicida Kumulus DF foi levemente nocivo (classe 2), ocasionando redução no parasitismo de $68,50 \%$. Os demais inseticidas não ocasionaram efeitos deletérios acima de $25,52 \%$ no parasitismo de $T$. pretiosum, sendo todos considerados inócuos (classe 1) (Tabela 1). No Bioensaio $\mathrm{V}$, realizado aos 31 DAP, também houve diferença significativa $(\mathrm{F}=8,26 ; \mathrm{gl}=5,18 ; \mathrm{p}=0,0003)$ entre os tratamentos. Da mesma forma que o bioensaio IV, apenas o fungicida Kumulus DF apresentou efeitos nocivos significativos ( $<<0,05$, Tukey-Kramer) sobre o parasitismo de T. pretiosum, ocasionando redução de $62,36 \%$ no número de ovos parasitados. Os demais inseticidas, Dipterex 500, Malathion $1000 \mathrm{CE}$, Sumithion $500 \mathrm{CE}$ e Tiomet $400 \mathrm{CE}$ não apresentaram diferenças significativas ( $p>0,05$, Tukey-Kramer) para o número de ovos parasitados em relação à testemunha do bioensaio. Os demais inseticidas continuaram classificados na classe inócuos (classe 1), ocasionando reduções no parasitismo de, no máximo, 16,99\% (Tabela 1). Na Tabela 2, são apresentadas as classes de persistência biológica da IOBC/WPRS para testes com artrópodes benéficos. Os inseticidas organofosforados
Dipterex 500, Sumithion 500 CE e Tiomet 400 CE foram levemente persistentes (classe 2), pois ocasionaram redução no parasitismo de T. pretiosum até 10 DAP (Tabela 1). Por outro lado, o inseticida Malathion $1000 \mathrm{CE}$ reduziu o parasitismo até os $17 \mathrm{DAP}$, sendo, portanto, considerado como moderadamente persistente (classe 3). A classe persistente (classe 4) foi atribuída apenas ao fungicida/ acaricida Kumulus DF, à base de enxofre, que reduziu constantemente o parasitismo (Tabela 1) de T. pretiosum até 31 DAP.

$\mathrm{Na}$ literatura, estão disponíveis resultados de testes de persistência biológica apenas com adultos da espécie padrão da IOBC/WPRS T. cacoeciae. Dessa forma, resultados similares aos obtidos na presente pesquisa foram observados para o fungicida/ acaricida Kumulus DF (Grützmacher et al., 2004). Por outro lado, distintas observações foram obtidas para os inseticidas dimetoato (Perfekthion) (Hassan et al., 1988), fenitrotiona (Folithion 550) (Hassan et al., 1987) e triclorfom (Dipterex 500) (Grützmacher et al., 2004), sendo que todos esses autores obtiveram a classe 4 (persistente) para esses agrotóxicos, pois ocasionaram reduções no número de ovos parasitados mesmo em períodos superiores a 31 DAP.

Os resultados distintos observados, provavelmente, devemse às diferentes condições ambientais em que foram realizados os estudos. Sabe-se que a persistência de um agrotóxico que se deposita na superfície das plantas está diretamente relacionada com processos degradativos bióticos e abióticos junto com a volatilização (Silva \& Fay, 2004). Essa tem uma forte dependência, normalmente não-linear, com a temperatura. Em função disso, são encontrados diferentes valores de volatilização para uma mesma molécula quando em diferentes condições climáticas (Silva \& Fay, 2004). O presente estudo foi conduzido com plantas cultivadas em casa de vegetação, sem controle de condições ambientais, e nos meses de janeiro a março, que se caracterizam por temperaturas máximas elevadas no $\operatorname{RS}\left(30,5^{\circ}\right.$ a $\left.32^{\circ}\right)$, o que, provavelmente, contribuiu para uma aceleração nos processos de degradação dos agrotóxicos. Em estudo de persistência realizado por Van de Veire et al. (2004), em diferentes países (Bélgica, Espanha e Itália), e com Encarsia formosa Gahan, 1924 (Hymenoptera: Aphelinidae), esses autores atribuíram os resultados discrepantes obtidos às diferentes intensidades de luz (fotodegradação), visto que a temperatura não diferiu significativamente entre os locais de estudo.

Além das condições ambientais, diferenças de suscetibilidade interespecífica e/ou dosagens distintas também influenciam na suscetibilidade aos agrotóxicos. A existência de diferenças de suscetibilidade interespecífica de Trichogramma spp. e intra-específica para $T$. pretiosum a agrotóxicos já foram previamente relatadas (Bull \& Coleman, 1985; Carvalho et al., 2003) e demonstram a necessidade de realização de estudos específicos com a espécie, se possível, com a linhagem a ser utilizada no programa de controle biológico da cultura-alvo. Com relação a distintas dosagens utilizadas, nos trabalhos de Hassan et al. (1988) com dimetoato e de Hassan et al. (1987) com fenitrotiona, foram utilizadas concentrações superiores desses ingredientes ativos, respectivamente, de 210 e $100 \mathrm{~g}$ i.a. $100 \mathrm{~L}^{-1}$, contra os 48 e 75 g i.a. $100 \mathrm{~L}^{-1}$, avaliados no presente estudo, o 
que, provavelmente, também contribuiu para a discrepância entre os resultados obtidos em ambos os estudos.

Testes de persistência biológica auxiliam na estimativa da toxicidade de um agrotóxico, pois o seu impacto no campo é bastante afetado pela sua persistência. Agrotóxicos de vida curta poderiam ser utilizados com freqüência em programas integrados de controle, na ausência de produtos inócuos. De acordo com a seqüência de testes da IOBC/WPRS (Hassan \& Abdelgader, 2001) para Trichogramma, os inseticidas Dipterex 500, Sumithion 500 CE e Tiomet $400 \mathrm{CE}$, classificados como levemente persistentes (classe 2), não necessitam prosseguir na seqüência de testes. Por outro lado, Malathion $1000 \mathrm{CE}$ e Kumulus DF, que foram

TABELA 1 - Número de ovos parasitados por fêmea, redução (\%) no parasitismo e classes da IOBC para agrotóxicos indicados na produção integrada de pêssego a adultos de Trichogramma pretiosum no bioensaio de persistência biológica. FAEM/ UFPel, 2005.

\begin{tabular}{|c|c|c|c|c|c|}
\hline $\begin{array}{l}\text { Produto } \\
\text { comercial }\end{array}$ & Ingrediente ativo & $\mathrm{DC}^{*}$ & $\begin{array}{c}\text { Ovos parasitados } \\
\text { por fêmea }^{* \star \star *}\end{array}$ & $\mathrm{RP}^{* * * * *}$ & $\mathrm{C}^{\mathrm{stat+2}}$ \\
\hline \multicolumn{6}{|c|}{ Bioensaio I - 03 DAP } \\
\hline & testemunha & & $43,27 \mathrm{a}$ & & \\
\hline Dipterex 500 & triclorfom & 300 & $0,30 \mathrm{~b}$ & 99,31 & 4 \\
\hline Kumulus $^{\sqrt{ }} \mathrm{DF}$ & enxofre & 600 & $1,48 \mathrm{~b}$ & 96,58 & 3 \\
\hline Malathion $1000 \mathrm{CE}$ & malationa & 200 & $0,00 \mathrm{~b}$ & 100,00 & 4 \\
\hline Sumithion $^{\circledR} 500 \mathrm{CE}$ & fenitrotiona & 150 & $10,81 \mathrm{~b}$ & 75,02 & 2 \\
\hline Tiomet $^{\mathbb{E}} 400 \mathrm{CE}$ & dimetoato & 120 & $0,00 \mathrm{~b}$ & 100,00 & 4 \\
\hline \multicolumn{6}{|c|}{ Bioensaio II - 10 DAP } \\
\hline & testemunha & - & $33,19 a$ & - & - \\
\hline Dipterex $^{8} 500$ & triclorfom & 300 & $16,86 \mathrm{~b}$ & 49,20 & 2 \\
\hline Kumulus ${ }^{\mathbb{8}} \mathrm{DF}$ & enxofre & 600 & $4,52 \mathrm{bc}$ & 86,38 & 3 \\
\hline Malathion $1000 \mathrm{CE}$ & malationa & 200 & $0,00 \mathrm{c}$ & 100,00 & 4 \\
\hline Sumithion $500 \mathrm{CE}$ & fenitrotiona & 150 & $18,25 \mathrm{ab}$ & 45,01 & 2 \\
\hline Tiomet $400 \mathrm{CE}$ & dimetoato & 120 & 14,48 bc & 56,37 & 2 \\
\hline \multicolumn{6}{|c|}{ Bioensaio III - 17 DAP } \\
\hline & testemunha & - & $38,40 \mathrm{a}$ & $-\cdots-$ & $\ldots$ \\
\hline Dipterex ${ }^{8} 500$ & triclorfom & 300 & $33,65 \mathrm{ab}$ & 12,37 & 1 \\
\hline Kumulus ${ }^{8} \mathrm{DF}$ & enxofre & 600 & $4,44 \mathrm{c}$ & 88,44 & 3 \\
\hline Malathion $1000 \mathrm{CE}$ & malationa & 200 & $3,01 \mathrm{c}$ & 92,16 & 3 \\
\hline Sumithion $^{\circledR} 500 \mathrm{CE}$ & fenitrotiona & 150 & $28,48 \mathrm{~b}$ & 25,83 & 1 \\
\hline Tiomet $^{8} 400 \mathrm{CE}$ & dimetoato & 120 & $40,21 \mathrm{a}$ & 0,00 & 1 \\
\hline \multicolumn{6}{|c|}{ Bioensaio IV - 24 DAP } \\
\hline & testemunha & - & $28,29( \pm 2,45) \mathrm{a}$ & - & - \\
\hline Dipterex 500 & triclorfom & 300 & $27,03( \pm 9,98)$ a & 4,45 & 1 \\
\hline Kumulus ${ }^{\mathbb{D}} \mathrm{DF}$ & enxofre & 600 & $8,91( \pm 3,08) b$ & 68,50 & 2 \\
\hline Malathion $1000 \mathrm{CE}$ & malationa & 200 & $22,09( \pm 4,65) \mathrm{ab}$ & 21,92 & 1 \\
\hline Sumithion $^{1} 500 \mathrm{CE}$ & fenitrotiona & 150 & $21,07( \pm 2,68) a b$ & 25,52 & 1 \\
\hline Tiomet $^{\mathbb{8}} 400 \mathrm{CE}$ & dimetoato & 120 & $24,37( \pm 5,51)$ a & 13,86 & 1 \\
\hline \multicolumn{6}{|c|}{ Bioensaio V - 31 DAP } \\
\hline & testemunha & - & $31,14( \pm 2,94)$ a & - & - \\
\hline Dipterex 500 & triclorfom & 300 & $33,03( \pm 5,93)$ a & 0,00 & 1 \\
\hline Kumulus ${ }^{8}$ DF & enxofre & 600 & $11,72( \pm 5,37) b$ & 62,36 & 2 \\
\hline Malathion $1000 \mathrm{CE}$ & malationa & 200 & $31,89( \pm 8,03)$ a & 0,00 & 1 \\
\hline Sumithion $500 \mathrm{CE}$ & fenitrotiona & 150 & $25,85( \pm 2,53)$ a & 16,99 & 1 \\
\hline Tiomet $^{\mathbb{E}} 400 \mathrm{CE}$ & dimetoato & 120 & $31,44( \pm 0,69)$ a & 0,00 & 1 \\
\hline
\end{tabular}

${ }^{*} \mathrm{DC}=$ Dosagem do produto comercial $\left(\mathrm{g}\right.$ ou $\left.\mathrm{mL} 100 \mathrm{~L}^{-1}\right)$.

${ }^{* *} \mathrm{DAP}=$ Dias após aplicação dos tratamentos

${ }^{* * * *}$ Médias seguidas por letras idênticas não diferem significativamente $(\mathrm{p}>0,05)$, pelo teste de (Kruskal-Wallis) Bonferroni-Dunn $t$ (Bioensaio I: $\mathrm{K}=20,65 ; \mathrm{p}=0,0009$; Bioensaio II: $\mathrm{K}=17,12 ; \mathrm{p}=0,0043$; Bioensaio III: $\mathrm{K}=18,05 ; \mathrm{p}=0,0029$ ) ou (ANOVA) Tukey-Kramer (Bioensaio IV: $\mathrm{F}=4,57$; $\mathrm{gl}=5,17 ; \mathrm{p}=0,0080$; Bioensaio $\mathrm{V}: \mathrm{F}=8,26 ; \mathrm{gl}=5,18 ; \mathrm{p}=0,0003$ ). Resultados expressam a média ( \pm erro-padrão) de 4 repetições.

${ }^{* * * *} \mathrm{RP}=$ Redução no parasitismo comparado com a testemunha.

$\mathrm{C}=$ classes da IOBC/WPRS para testes de seletividade em laboratório: $1=$ inócuo $(<30 \%), 2=$ levemente nocivo $(30-79 \%), 3=$ moderadamente nocivo $(80-99 \%), 4=$ nocivo $(>99 \%)$. classificados, respectivamente, como moderadamente persistente (classe 3) e persistente (classe 4), devem ser avaliados em campo, em pomares de pessegueiro.

TABELA 2 - Duração (dias) da atividade nociva (persistência biológica) de agrotóxicos indicados na produção integrada de pêssego a adultos de Trichogramma pretiosum e classes da IOBC/WPRS para bioensaios de persistência biológica. FAEM/ UFPel, 2005.

\begin{tabular}{|c|c|c|c|c|}
\hline \multirow{2}{*}{ Produto comercial } & \multirow{2}{*}{ Ingrediente ativo } & \multirow{2}{*}{$\mathbf{D C}^{*}$} & \multicolumn{2}{|c|}{ Persistência biológica } \\
\hline & & & Dias & $\mathrm{C}^{*}$ \\
\hline Dipterex $^{8} 500$ & triclorfom & 300 & $>10-<17$ & 2 \\
\hline Kumulus ${ }^{\circledR}$ DF & enxofre & 600 & $>31$ & 4 \\
\hline Malathion ${ }^{\circledR} 1000 \mathrm{CE}$ & malationa & 200 & $>17-<24$ & 3 \\
\hline Sumithion ${ }^{\circledR} 500 \mathrm{CE}$ & fenitrotiona & 150 & $>10-<17$ & 2 \\
\hline Tiomet $^{\circledR} 400 \mathrm{CE}$ & dimetoato & 120 & $>10-<17$ & 2 \\
\hline
\end{tabular}

${ }^{*} \mathrm{DC}=$ Dosagem do produto comercial $\left(\mathrm{g}\right.$ ou $\left.\mathrm{mL} 100 \mathrm{~L}^{-1}\right)$.

${ }^{* *} \mathrm{C}=$ classes da IOBC/WPRS, para testes de persistência biológica: 1=vida curta ( $<5$ dias); $2=$ levemente persistente $(5-15$ dias $) ; 3=$ moderadamente persistente (16-30 dias); $4=$ persistente ( $>30$ dias).

\section{CONCLUSÃO}

Para os produtos comerciais, nas dosagens ( $\mathrm{g}$ ou $\mathrm{mL} 100$ $\left.\mathrm{L}^{-1}\right)$ avaliadas e nas condições em que foram realizados os bioensaios, conclui-se que: os inseticidas Dipterex 500 (300), Sumithion 500 CE (150) e Tiomet 400 CE (120) são levemente persistentes (5-15 dias de ação nociva), Malathion 1000 CE (200) é moderadamente persistente (16-30 dias de ação nociva) e o fungicida/acaricida Kumulus DF (600) é persistente ( $>31$ dias de ação nociva).

\section{REFERÊNCIAS}

BOTTON, M.; ARIOLI, C.J.; COLLETTA, V.D. Monitoramento da mariposa oriental Grapholita molesta (Busck, 1916) (Lepidoptera: Tortricidae) na cultura do pessegueiro. Bento Gonçalves: Embrapa CNPUV, 2001. 4p. (Comunicado Técnico, 38)

BULL, D.L.; COLEMAN, R.J. Effects of pesticides on Trichogramma spp. Southwestern Entomologist, Dallas, v.8, p.156-168. 1985.

CARVALHO, G.A.; PARRA, J.R.P.; BAPTISTA, G.C. Bioatividade de produtos fitossanitários utilizados na cultura do tomateiro (Lycopersicum esculentum Mill.) a Trichogramma pretiosum Riley, 1879 (Hymenoptera: Trichogrammatidae) nas gerações $F_{1}$ $\mathrm{eF}_{2}$. Ciência e Agrotecnologia, Lavras, v.27, n.2, p.261-270, 2003.

CONOVER, W.J.; IMAN, R.L. Rank transformations as a bridge between parametric and nonparametric statistics. The American Statistician, Alexandria, v.35, n.3, p.124-129, 1981. 
FACHINELLO, J.C.; TIBOLA, C.S.; VICENZI, M.; PARISOTO, E.; PICOLOTTO, L.; MATTOS, M.L.T. Produção integrada de pêssegos: três anos de experiência na região de Pelotas-RS. Revista Brasileira de Fruticultura, Jaboticabal, v.25, n.2, p.256258,2003

GARCIA, M.S.; SILVA, W.D.; ZAZYCKI, L.C.F.; BOTTON, M.; PARRA, J.R.P. Determinação do número de Trichogramma pretiosum a ser liberado por ovo de Grapholita molesta em condições de semicampo. In: CONGRESSO BRASILEIRO DE ENTOMOLOGIA, 21., 2006, Recife. Resumos... Pernambuco: SEB, 2006. CD-ROM.

GIOLO, F.P.; GRÜTZMACHER, A.D.; MANZONI, C.G.; FACHINELLO, J.C; NÖRNBERG, S.D.; STEFANELLO JÚNIOR, G.J. Seletividade de agrotóxicos indicados na produção integrada de pêssego a Trichogramma pretiosum (Hymenoptera: Trichogrammatidae). Revista Brasileira de Fruticultura, Jaboticabal, v.27, n.2, p. 222-225, 2005.

GRÜTZMACHER, A.D.; ZIMMERMANN, O.; YOUSEF, A.; HASSAN, S.A. The side-effects of pesticides used in integrated production of peaches in Brazil on the egg parasitoid Trichogramma cacoeciae Marchal (Hym., Trichogrammatidae). Journal of Applied Entomology, Berlin, v.128, n.6, p.377-383, 2004.

HASSAN, S.A. The suitability of Trichogramma cacoeciae as an indicator species for testing the side effect of pesticides on beneficial arthropods, compared to other parasitoids. IOBC/ WPRS Bulletin, Montfavet, v.21, n.6, p.89-92, 1998.

HASSAN, S.A.; ABDELGADER, H. A sequential testing program to assess the effects of pesticides on Trichogramma cacoeciae Marchal (Hym., Trichogrammatidae). IOBC/WPRS Bulletin, Montfavet, v.24, n.4, p.71-81, 2001.
HASSAN, S.A.; ALBERT, R.; BIGLER, F.; BLAISINGER, P.; BOGENSCHÜTZ, H.; BOLLER, E.; BRUN, J.; CHIVERTON, P.; EDWARDS, P.J.; ENGLERT, W.D.; HUANG, P.; INGLESFIELD, C.; NATON, E.; OOMEN, P.A.; OVERMEER, W.P.J.; RIECKMANN, W.; SAMSOE-PETERSEN, L.; STÄUBLI, A.; TUSET, J.J.; VIGGIANI, G.; VANWETSWINKEL, G. Results of the third joint pesticide testing programme by the IOBC/WPRS Working Group "Pesticides and Beneficial Organisms". Zeitschrift für Angewandte Entomologie, Berlin, v.103, p.92-107, 1987.

HASSAN, S.A.; BIGLER, F.; BOGENSCHÜTZ, H.; BOLLER, E.; BRUN, J.; CHIVERTON, P.; EDWARDS, P.J.; MANSOUR, F.; NATON, E.; OOMEN, P.A.; OVERMEER, W.P.J.; POLGAR, L.; RIECKMANN, W.; SAMSOE-PETERSEN, L.; STÄUBLI, A.; STERK, G.; TAVARES, K.; TUSET, J.J.; VIGGIANI, G.; VIVAS, A.G. Results of the fourth joint pesticide testing programme carried out by the IOBC/WPRS - Working Group "Pesticides and Beneficial Organisms". Zeitschrift für Angewandte Entomologie, Berlin, v.105, p.321-329, 1988.

SAS Institute. Getting Started with the SAS Learning Edition. Cary, NC: SAS Institute, 2002.

SILVA, C.M.S.; FAY, E.F. Agrotóxicos e ambiente. Brasília: Embrapa Informação Tecnológica, 2004. 400p.

VAN DE VEIRE, M.; VIÑUELA, E.; BERNARDO, U.; TIRRY, L.; ADAN, A.; VIGGIANI, G. Duration of the toxicity of abamectin and spinosad on the parasitic wasp Encarsia formosa Gahan in northern and Southern Europe. IOBC/WPRS Bulletin, Montfavet, v.27, n.6, p.21-30, 2004. 